\title{
DOSAGE DE L'AZOTE AMINÉ DANS QUELQUES LIQUIDES BIOLOGIQUES
}

\author{
M. C. MICHEL \\ Avec la collaboration technique de Janine WaGNir. \\ Service de Biochimie et de Nutrition, \\ Centre national de Recherches zootechniques, Jouy-en-Josas.
}

\section{SOMMAIRE;}

$I^{\circ}$ Les substances qui possèdent un groupement aminé libre en alpha d'un carboxyle sont dégradées par la ninhydrine avec formation d'ammoniac, qui est ensuite. dosé après microdiffusion par la méthode de ConWAY.

$2^{\circ}$ Les acides aminés, dosés par cette méthode, foumissent la quantité théorique d'ammoniac, sauf la proline, le tryptophane et l'acide aspartique, qui donnent des résultats plus faibles.

$3^{\circ}$ L'azote aminé contenu dans le sang, les contenus intestinaux du porc, a été dosé par cette méthode.

$4^{0}$ Divers procédés de déprotéinisation ont été étudiés et spécialement l'emploi d'alcool éthylique.

$5^{\circ}$ La comparaison de cette méthode avec celle de VAN SLYKE à l'acide nitreux montre que pour les acides aminés, cette dernière fournit des résultats voisins de la théorie sauf pour la lysine (I95 p. roo) et la proline (o).

En revanche, la mesure par VAN SLYKE de l'azote aminé du sang et de contenus de l'intestin grêle du porc, donne des résultats nettement plus élevés que ceux obterus par la méthode à la ninhydrine.

\section{IN'TRODUC'TION}

La détermination quantitative des acides aminés libres est importante pour l'étude biochimique du métabolisme azoté. Il s'agit en particulier de mesurer leur concentration, en vue d'études de leur disponibilité au niveau intestinal, dans le sang porte, ou encore la quantité excrétée dans l'urine.

La teneur en acides aminés libres de ces différents milieux est généralement faible par rapport à d'autres substances susceptibles d'interférer dans leur dosage. Le sang, par exemple, contient environ $0,06 \mathrm{~g}$ par litre de $\mathrm{N}$ aminé (Hamirton et VAN SLYKE I943), alors que sa teneur en azote total est de l'ordre de 25 à $30 \mathrm{~g} / 1$.

La mesure de l'azote aminé peut fournir une bonne approximation de la teneur en acides aminés si la spécificité de la méthode employée est suffisante. De nombreux procédés ne remplissent pas cette condition. Il s'agit en partictulier de la titrimétrie, par la méthode de SoRENSEN (I907) et de ses modifications analysées par ScHMIDT (I938) et TAYLOR (I957), de la méthode de VAN SLyKE (I933) à l'acide nitreux et du dosage au cuivre de Pope et Stevens (I939).

Dans toutes ces méthodes, on dose les groupements aminés libres des acides aminés mais également ceux des peptides, protéines, et tout ou partie des aminés aliphatiques. L'urée et 1'ammoniac interfèrent également (VAN SLYKE et al I943aIRVING et al I944 $a$ - $b$ ) 
L'utilisation de la ninhydrine, par contre, permet une mesure plus spécifique. On sait que cette substance réagit avec un groupement aminé placé en alpha d'un carboxyle pour donner une aldéhyde, du $\mathrm{CO}_{2}$ et $\mathrm{NH}_{3}$. La mesure du $\mathrm{CO}_{2}$ formé a été mise au point par VAN SLyke et al (I938) (I94I) (I943) et Hamiron et VAN SLYKe (I943). La spécificité de ce dosage a été améliorée par SHotT et al (I944) et leurs résultats ont été confirmés par HaMIL'TON et VAN SLYKE (I946). Dans cette méthode, l'urée interfère et doit être éliminée. De plus 1'appareillage nécessaire se prête mal à des mesures en série. MAC FADYEN (I944) et SOBEI, et al (I946) ont décrit une méthode basée sur la dégradation du groupement aminé par la ninhydrine. Par ébullition en milieu acide on obtient un complexe de la ninhydrine avec l'ammoniac obtenu. Le $\mathrm{NH}_{3}$ est ensuite libéré par le perhydrol, déplacé par aération en milieu alcalin et titré dans l'acide borique.

La méthode que nous avons mise au point représente une simplication de ces deux dernières, destinées à effectuer un grand nombre de mesures avec un appareillage simple; le groupement aminé est dégradé par la ninhydrine, après déprotéinisation si nécessaire. L'ammoniac formé est titré après microdiffusion dans des cellules de ConwAY.

\section{MATÉRIEL, ETT MÉTHODES}

\section{A. - REACTIFS}

Tampon de pH 2.35:

Solution à 10 p. Ioo (Poids-volume), d'un mélange solide de :

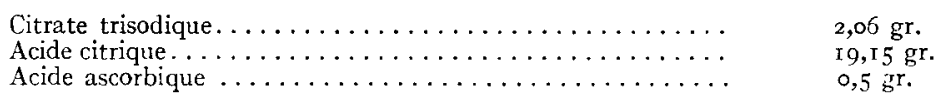

Perhydrol 100 volumes.

Ninhydrine.

$K O H$ saturée, environ $\mathrm{I} .100 \mathrm{~g} / \mathrm{l}$.

Solution d'acide borique :

Io $\mathrm{g}$ d'acide borique.

$200 \mathrm{ml}$ alcool à $95^{\circ}$.

Io $\mathrm{ml}$ indicateur (vert de bromocrésol $0,033 \mathrm{p}$. 100.

(rouge de méthyle $0,066 \mathrm{p}$. Ioo.

$\mathrm{H}_{2} \mathrm{O}$ q. s. I $000 \mathrm{ml}$. (dans l'alcool.

Acide chlorhydrique $\mathrm{N} / 70$.

Solution étalon contenant $200 \mathrm{mcg}$ de $\mathrm{N}(\mathrm{NH} 3)$ par $\mathrm{ml}$.

\section{B. - TECHNIQUE EXPERIMENTALE}

Extraction des acides aminés.

Le sang est prélevé dès l'abattage (sang artériel) ou à l'aide d'une canule posée dans la veineportc. Il est hémolysé par congélation. Le contenu intestinal est prélevé dès l'abattage, à divers niveaux du tractus. La déproténisation de ces échantillons est effectuée soit par! 'emploi d'alcool éthylique, soit par acidification :

Io Déprotéinisation par l'alcool éthylique. - Une partie aliquote $(20 \mathrm{~g})$ est diluée au $\mathrm{I} / \mathrm{1} / \mathrm{dans}$ l'alcool éthylique. La concentration utilisée, suivant les essais, varie de 80 à $90^{\circ}$, la concentration de $90 \mathrm{p}$. 10o étant finalement retenue. Le précipité est filtré et le filtre rincé par l'alcool de concentration correspondante. Le filtrat est évaporé à sec et le résidu repris par du tampon de $\mathrm{pH} \mathbf{2 , 3 5}$. On ramène à la concentration initiale pour le sang et on dilue au I/Io pour les contenus intestinaux.

$2^{\circ}$ Déprotéinisation par acidification. - L'échantillon de contenu intestinal $(20 \mathrm{~g})$ est acidifié par un volume d'acide trichloracétique à ıo p. Ioo ou sulfurique $2 \mathrm{~N}$. Le précipité obtenu est filtré sur Buchner et rincé par du tampon citrate de $\mathrm{pH} 2,35$. Le volume est amené à $200 \mathrm{ml}$ ave ce tampon. 


\section{C. - DOSAGE}

La solution à doser est diluée dans le tampon de manière à porter la concentration en azote aminé à environ $\mathrm{M} / \mathrm{ioo}$ et le $\mathrm{pH}$ de 2,4 à 2,6 .

$\mathrm{La}$ dégradation par la ninhydrine (50 $\mathrm{mg}$ ) s'ffectue dans des tubes de $56 / 160 \mathrm{~mm}$ contenant chacun $2,5 \mathrm{ml}$ de solution à doser (en double exemplaire). Les tubes placés dans un bain-marie bouillant, sont agités après I minute et laissés I 5 minutes. Après l'addition de $0,5 \mathrm{~cm}^{3}$ d'eau oxygénée, ils sont portés à nouveau 5 minutes au bain-marie.

Deux étalons sont préparés dans les mêmes conditions que les échantillons ; après refroidissement, deux prises aliquotes de I ml sont prélevées dans chaque tube. On dose selon Conway, en utilisant la potasse saturée pour déplacer l'ammoniac. La diffusion s'effectue en 2 heures à la température ambiante, et le titrage par l'acide chlorhydrique $\mathrm{N} / 7 \mathrm{O}$.

\section{RÉSULTATS OBTENUS}

I. - Des solutions d'acides aminés, d'amides et de peptides à la concentration M/Ioo ont été dosées par la méthode décrite ; les résultats ont été comparés à ceux obtenus par le procédé VAN SLyke à l'acide nitreux (tableau r).

TABLEAU I

Dosage de l'azote aminé de diverses substances. - Pourcentages oblenus par rapport à un $\mathrm{NH}^{2}$.

\begin{tabular}{|c|c|c|}
\hline & $\begin{array}{l}\text { Méthode décrite : } \\
\text { Ninhydrine - } \mathrm{NH}_{3} \\
\text { Conway }\end{array}$ & $\begin{array}{c}\text { Méthode } \\
\text { VAX SLYKE } \\
\mathrm{N}_{2}\end{array}$ \\
\hline Glycine $\ldots \ldots \ldots \ldots \ldots$ & 98 & $10^{\prime}$ \\
\hline 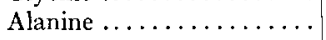 & 99,9 & 99 \\
\hline Sérine ............... & 101,5 & 103 \\
\hline Thréonine ............. & 98,6 & 105 \\
\hline Valine ............... & 97,5 & 104 \\
\hline Leucine .............. & 97,6 & 101 \\
\hline Isoleucine ............ & 98,4 & 100 \\
\hline Acide aspartique ....... & 89,1 & 92 \\
\hline Acide glutamique...$\ldots$ & 99,5 & 100 \\
\hline Arginine $\ldots \ldots \ldots \ldots \ldots$ & 102,5 & 100 \\
\hline Lysine ............... & $10 \frac{1}{2}, 2$ & 195 \\
\hline Cystéine ............. & 66,3 & 128 \\
\hline Méthionine ............ & 94,6 & 101,8 \\
\hline Phényl-alanine .......... & 96,2 & 101 \\
\hline Tyrosine .............. & 100,8 & $10^{\prime}, 7$ \\
\hline Tryptophane .......... & 66,3 & $100^{\prime}$ \\
\hline Histidine...$\ldots \ldots \ldots$ & 117,6 & 100 \\
\hline Proline .............. & 21 & 0 \\
\hline$\beta$ alanine $\ldots \ldots \ldots \ldots \ldots$ & 0 & 100 \\
\hline Urée $\ldots \ldots \ldots \ldots \ldots \ldots$ & 0 & 33 \\
\hline Asparagine ............ & 126,5 & 101 \\
\hline Glutamine ............ & 117,8 & 178 \\
\hline Glycyl-glycine ......... & 97 & $13 \prime_{t}^{\prime}$ \\
\hline Glycyl-L-leucine . ......... & 95 & 146 \\
\hline Glycyl-L-tyrosine . . . . . . & 79 & 122 \\
\hline Di-glycyl-glycine.......... & 94 & 121 \\
\hline dl-leucyl-glycine $\ldots \ldots \ldots$ & 0 & 128 \\
\hline dl-leucyl-glycyl-glycine ... & 0 & 107 \\
\hline 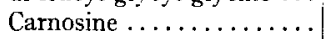 & 0 & 105 \\
\hline Histidyl-histidine ........ & 82 & 90 \\
\hline Glutathion ........... & 121 & 199 \\
\hline
\end{tabular}


II. Comparaison de divers modes d'extraction. - La déprotéinisation à l'alcool éthylique présente divers avantages. Les protéines sont précipitées à partir d'une solution neutre, à basse température, ce qui limite au maximum la dégradation des

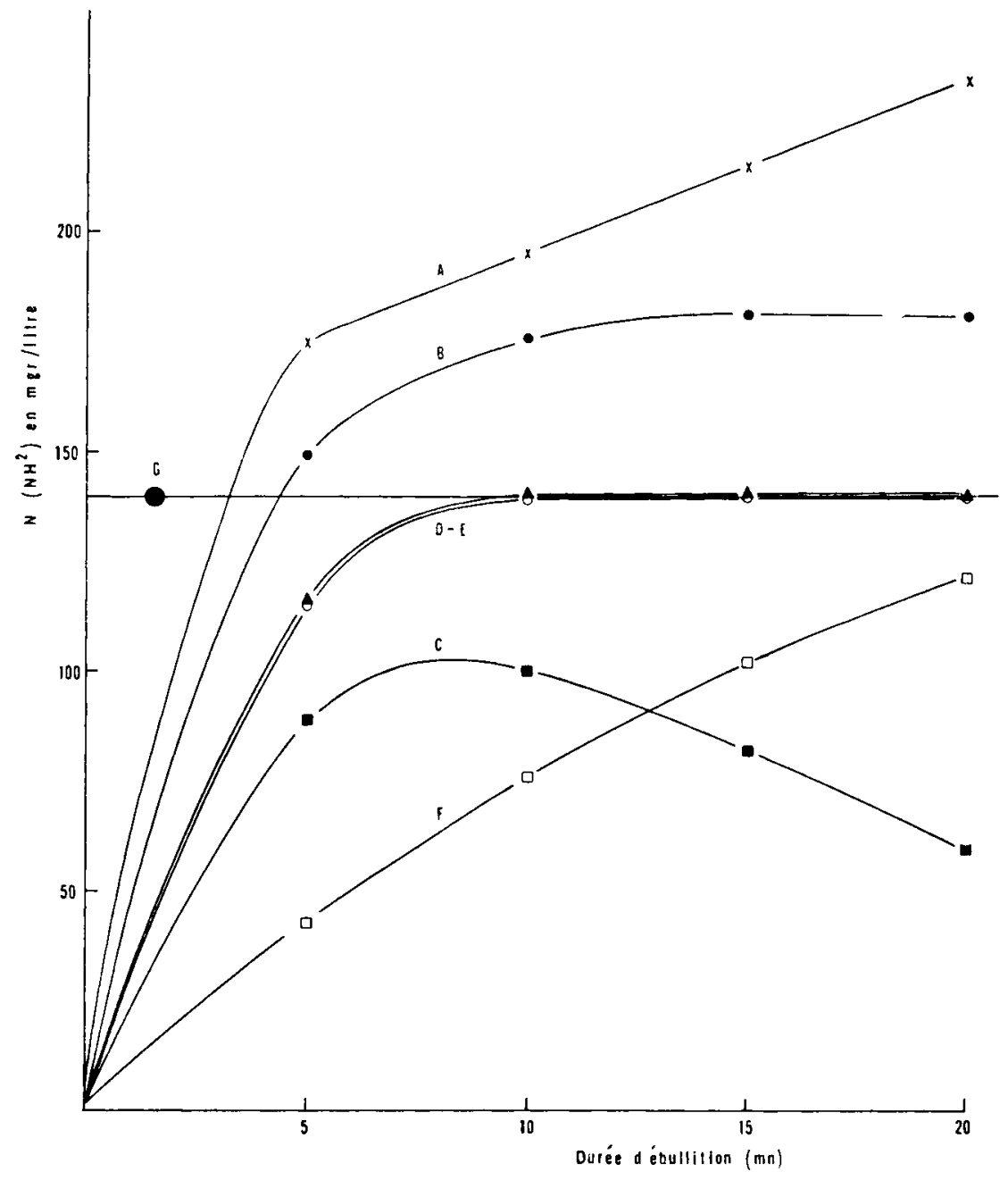

FIG. I.

$\times$ A Extraction du contenu intestinal par $\mathrm{H}_{2} \mathrm{SO}_{4}$.

B Extraction du contenu intestinal par alcool à $90^{\circ}$.

C Extraction du contenu intestinal par acide trichloracétique.

A D Solution standard : Alanine $\mathrm{M} / \mathrm{I}$ oo dans le tampon citrate.

$\bigcirc \mathrm{E}$ Solution standard : Alanine $\mathrm{M} / \mathrm{I} 00$ dans $\mathrm{H}_{2} \mathrm{SO}_{4}$.

$\mathrm{F}$ Solution standard : Alanine $\mathrm{M} / \mathrm{r} 00$ dans acide trichloracétique

G Teneur théorique de la solution standard.

substances labiles, telles que la glutamine du sang. La dégradation enzymatique des substances azotées, qui continue à s'exercer dans des contenus intestinaux maintenus à température ambiante, est également limitée au maximum. Enfin la remise en solution de résidu obtenu par séchage de l'extrait alcoolique peut être effectuée dans 
un petit volume, de manière à pouvoir doser des solutions dont la teneur en azote aminé est faible au départ.

D'autres agents de déprotéinisation sont couramment utilisés, tel que l'acide trichloracétique. Les essais suivants ont pour but de comparer les teneurs en azote aminé obtenues après déprotéinisation de contenus intestinaux par les acides trichloracétique et sulfurique, et par l'alcool éthylique à différentes concentrations.

La figure I indique l'influence de l'agent d'extraction sur la cinétique du dosage par la ninhydrine de l'azote aminé d'un contenu intestinal de porc. Une solution d'alanine $\mathrm{M} /$ IOo, traitée de la même manière, a été utilisée comme solution standard.

III. Comparaison des méthodes à la ninhydrine et à l'acide nitreux pour le dosage de l'azote aminé dans le sang et les contenus intestinaux du porc.

a) Dans cette série d'essais, reportée dans le tableau $2,1^{\prime}$ alcool à $85^{\circ}$ a été utilisée pour la déprotéinisation.

TABLEAU 2

Teneur en $\mathrm{N}\left(\mathrm{NH}_{2}\right)$ des contenus du tractus gastro-intestinal et du sang artériel du porc. (résultats exprimés en mg/litre).

\begin{tabular}{|c|c|c|}
\hline $\begin{array}{l}\text { Échantillons extraits } \\
\text { par l'alcool à } 85^{\circ} \\
\text { Porcs à jeun }\end{array}$ & $\begin{array}{l}\text { Méthode décrite : } \\
\text { Ninhydrine-NH } \\
\text { Conway }\end{array}$ & $\begin{array}{c}\text { Méthode } \\
\text { VAN SLYKE } \\
\mathrm{N}_{2}\end{array}$ \\
\hline Estomac $\left({ }^{1}\right)\left({ }^{2}\right) \ldots \ldots \ldots$ & $\begin{array}{l}482 \\
512 \\
420 \\
356\end{array}$ & $\begin{array}{l}500 \\
570 \\
477 \\
389\end{array}$ \\
\hline Jejunum $\ldots \ldots \ldots \ldots \ldots$ & $\begin{array}{r}1360 \\
1190 \\
1428 \\
926\end{array}$ & $\begin{array}{r}1796 \\
2212 \\
2533 \\
948\end{array}$ \\
\hline Iléon $\ldots \ldots \ldots \ldots \ldots \ldots$ & $\begin{array}{l}572 \\
844 \\
725 \\
650\end{array}$ & $\begin{array}{r}783 \\
1125 \\
1010 \\
820\end{array}$ \\
\hline Sang périphérique total .. & $\begin{array}{l}79 \\
74 \\
78 \\
73 \\
74 \\
87 \\
95\end{array}$ & $\begin{array}{l}117\left(^{(3}\right) \\
109 \\
115 \\
115 \\
120 \\
100 \\
107\end{array}$ \\
\hline \multicolumn{3}{|c|}{$\begin{array}{l}\text { (1) Chacune de ces mesures est effectuée sur un échantillon différent. } \\
\left({ }^{2}\right) \text { Dans le cas où les substances à doser contiennent des sels ammonia- } \\
\text { caux, on effectue un dosage direct sans ninhydrine ni ébullition. } \\
\left({ }^{3}\right) \text { La correction due à l'urée n'a pas été effectuée. Celle-ci apporte de } 7 \\
\text { à } 15 \mathrm{mg} / \text { litre dans ces résultats. }\end{array}$} \\
\hline
\end{tabular}

b) Influence de la concentration de l'alcool éthylique utilisé pour la déprotéinisation sur la teneur en azote aminé du sang et de contenus intestinaux (Tableau 3 ).

c) Ces résultats indiquent que 1'alcool éthylique à $80^{\circ}$ solubilise, outre des acides aminés libres, des substances susceptibles d'être dosées par l'acide nitreux. L'extrait 
TABLEAU 3

Déprotéinisation par l'alcool à différentes concentrations.

(Teneurs en $\left.\mathrm{N}\left(\mathrm{NH}_{2}\right) \mathrm{mg} / \mathrm{l}\right)$.

\begin{tabular}{|c|c|c|c|}
\hline $\begin{array}{l}\text { Concentration } \\
\text { alcoolique }\end{array}$ & Méthode décrite & $\begin{array}{c}\text { VAN SLikk } \\
\text { nitreux }\end{array}$ & $\begin{array}{c}\text { Nature } \\
\text { de l'échantillon }\end{array}$ \\
\hline $\begin{array}{l}80^{\circ} \\
85^{\circ} \\
90^{\circ}\end{array}$ & $\begin{array}{l}79,8 \\
76,8 \\
75,9\end{array}$ & $\begin{array}{r}112,2 \\
100,5 \\
90,2\end{array}$ & $\begin{array}{l}\text { Sang artériel du } \\
\text { porc. }\end{array}$ \\
\hline $\begin{array}{l}80^{\circ} \\
85^{\circ} \\
90^{\circ}\end{array}$ & $\begin{array}{l}13 / 0 \\
1280 \\
1080\end{array}$ & $\begin{array}{l}2750 \\
2150 \\
1460\end{array}$ & $\begin{array}{l}\text { Conteruu du jéju- } \\
\text { num. }\end{array}$ \\
\hline $\begin{array}{l}80^{\circ} \\
85^{\circ} \\
90^{\circ}\end{array}$ & $\begin{array}{l}600 \\
570 \\
530\end{array}$ & $\begin{array}{r}1560 \\
1320 \\
770\end{array}$ & Contenu de l'iléon. \\
\hline
\end{tabular}

alcoolique contient en effet une forte proportion d'azote total non protéique, comparée au $\mathrm{N}\left(\mathrm{NH}_{2}\right)$ dosable par la ninhydrine. Cette proportion diminue notablement lorsque le degré alcoolique s'accroît de 80 à $90^{\circ}$. Le tableau 4 résume les résultats obtenus sur le contenu du jéjunum de différents porcs.

TABI,EAU 4

Teneur en $N$ total et $N\left(\mathrm{NH}_{2}\right)$ de l'extrait alcoolique du contenu intestinal du porc.

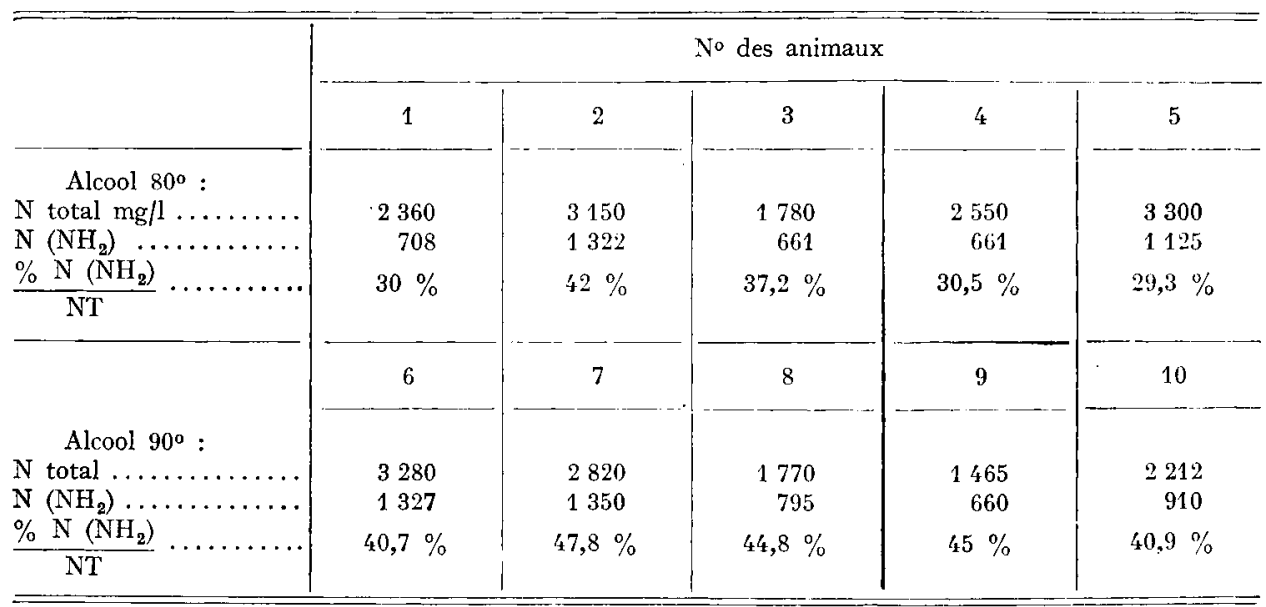

IV. Dosage d'acides aminés purs en surcharge dans des milieux biologiques. L'addition de solutions d'acides aminés purs à des échantillons de contenus intestinaux, avant la déprotéinisation par l'alcool à $90^{\circ}$, a pour but de vérifier le rendement de l'extraction (tableau 5). 
TABI,EAU 5

Surcharge d'acides aminés purs dans des contenus intesinaux.

\begin{tabular}{|c|c|c|c|c|}
\hline & \multicolumn{2}{|c|}{ Azote aminé mg/l } & \multirow{2}{*}{$\%$ retrouvé } & \multirow{2}{*}{$\begin{array}{c}\text { Nombre } \\
\text { d'observations }\end{array}$} \\
\hline & ajouté & $\begin{array}{l}\text { dosé avant } \\
\text { surcharge }\end{array}$ & & \\
\hline Alanine ... . & 135 & 585 & 99,8 上 1 & 5 \\
\hline Acide glutanique.... & id. & 1020 & $100,1 \pm 0,9$ & 5 \\
\hline Mélange standard*... & iil. & 1675 & $10^{2}, 5 \pm 3$ & 5 \\
\hline
\end{tabular}

* Le mélange standard contient les acides aminés du tableau I, à l'exception de la tyrosine et de la $\beta$-alanine. La teneur en azote aminé obtenue par dosage direct est de $96,4 \%$ de la théorie.

\section{DISCUSSION}

Ces résultats indiquent que la méthode à la ninhydrine fournit, pour la presque totalité des acides aminés étudiés, des résultats voisins de la valeur théorique. La proline et le tryptophane, et à un moindre degré l'acide aspartique, donnent des résultats plus faibles. L'ensemble de ces données est en bon accord avec les résultats de Sober et al (I945).

Les peptides dégradés sont ceux qui sont labiles dans les conditions du dosage (glutathion par exemple), et les glycyl-peptides.

La méthode de VAN SLYKE à l'acide nitreux fournit des résultats plus élevés que la théorie pour certains acides aminés (lysine en particulier) ; les peptides donnent également des résultats plus élevés que la méthode à la ninhydrine. L'urée est dégradée en partie. Ces différences entre les deux méthodes se retrouvent dans le dosage de l'azote aminé du sang et des contenus de l'intestin grêle du porc. Seul le contenu stomacal fournit des résultats assez concordants.

Le mode de déprotéinisation des milieux biologiques étudiés influe sur la teneur en azote aminé déterminé par les deux méthodes. En effet par 1'emploi d'alcool éthylique à diverses concentrations, des substances susceptibles d'êtrędosées par l'acide nitreux, et à un moindre degré par la ninhydrine, sont extraites lorsque le titre alcoolique décroit de $90^{\circ}$ à $80^{\circ}$. La déprotéinisation en milieu acide $\left(\mathrm{H}_{2} \mathrm{SO}_{4}\right)$, solubilise plus de substances dosables par la ninhydrine que par l'emploi d'alcool à $90^{\circ}$. D'après la cinétique du dosage, il semble qu'on se trouve plutôt en présence d'une hydrolyse de substances labiles dans ces conditions expérimentales (graphique Icourbe A). En présence d'acide trichloracétique, on observe des pertes d'ammoniac (courbe $c$ ), et la vitesse de dégradation du groupement aminé est ralentie (courbe $\mathbf{F}$ ).

En conclusion, la déprotéinisation des milieux biologiques par l'alcool éthylique, suivie du dosage par la méthode décrite, permet une estimation rapide et quantitative de l'azote aminé. 


\section{SUMMARY}

\section{ESTIMATION OF AMINO NITROGEN IN SOME HHOLOGICAL FLUIDS.}

I) Substances possessing a free amino group in the alpha position of a carboxyl are broken down by ninhydrin with the formation of ammonia, which is then estimated, after microdiffusion, by CONWAY's method.

2) The amino acids, determined by this method, supply the theoretical amount of ammonia, except for proline (2I p. I00), tryptophan and cysteine (66 p. I00) and aspartic acid (90 p. 100). Of the peptides, only those react which are labile under the conditions of the determination (glutathion I 2 I P. I oo) and the glycyl peptides. (table $\mathrm{I}$ ).

3) The amino nitrogen present in the blood and in the intestinal contents of the pig, has been estimated by this method, after protein removal with ethyl alcohol.

4) In comparison with the use of ethyl alcohol, the removal of protein by sulphuric acid at pII 2 gives higher values, because of the extraction of labile substances under the conditions of the determination (figure I A.) Trichloroacetic acid interferes with the action the ninhydrin and gives lower values (figure I $\mathrm{C}$ ).

5) The comparison of the method described with that of VAN SLYKE using nitrous acid shows that, for the amino acids, the latter method gives values close to the theoretical ones, except for lysine (195 p. 100) and proline (o p. 100). On the other hand, the VAN SLYKE method of measuring amino nitrogen of the blood and the contents of the small intestine of the pig gives distinctly higher values than those obtained by the ninhydrin method. Since the amino acid added to the biological material studied are quantitatively recovered by the ninhydrin method, it is probable that the differences observed between the two methods are due to the determination, by VAN SLYsEs'method, of substances other than free amino acids.

\section{RÉFÉRENCES BIBLIOGRAPHIQUES}

Hamitron P. B., VAN SLYKe D. D., Ig43. The gasometric determination of frec amino acids in blood filtrates by the ninhydrine carbon dioxide method. J. Biol. Chem., 150, 231

Hamiton P. B., VAN SLYKE D. D., ig46. The effects of the volatile adehydes formed on the accuracy of the manometric ninhydrin carbon dioxide method in analysis of certain alpha-amino arids. J. Biol. Chem., 164, 249 .

IRVING G. W., Fontaine T. D., SAMUeLS C. S., I944a. The reaction of ammonia in the VAN SIYKE volumetric amino nitrogen method. Arch. Biol., 4, 347.

IRVing G. W., FonTAINE T. D., SAmUeis C.S., 1944 b. Effect of light on the reaction of ammonia in the VAN SLYKe volumetric aminonitrogen method. Arch. Biochem., 4, 455.

MAC FADYEN D. A., I944. Determination of ammonia evolved from alpha-aminoacids by ninhydrin $J$. Biol. Chem., 153, 507 .

Pope C. G., Stevens M. F., 1939. The determination of amino nitrogen using a copper method. Biochem. J., 33, 1070.

Schmid' C. L. A., I938. The rhemistry of the amino acids and proteins. Thomas Springfield.

ShotT H. F., Rockland I. B., DUNN M. S., I944. A source of error in the manometric ninhydrin method for the analysis of amino acids and its suppression by use of hydrazine. J. Biol. Chem., 154, 397 .

Soble A. F., Hirshman A., Besmax L., 1945. A convenient microtitration method for the estimation of amino acids. J. Biol. Chem., 161, 99 .

TAYLOR W. H., I957. Formol titration: an evaluation of its various modifications. Analyst, 82, 488.

VAN SI,YKE D. D., Dillon R. T., 1938. Gasometric determination of carboxyl groups in free anino acids. J. Biol. Chem., 141, 627.

Van Slyke D. D., Mac Fadyen I. A., Hamilton P. B., 1943a. The gasometric determination of amino acids in urine by the ninhydrine carbon dioxide method. J. Biol. Chem., 150, 25 I.

VAN SLYKE D. D., I 929 . Manometric determination of primary amino nitrogen and its application to blood analysis. J. Biol. Chem., 83, 425 . 